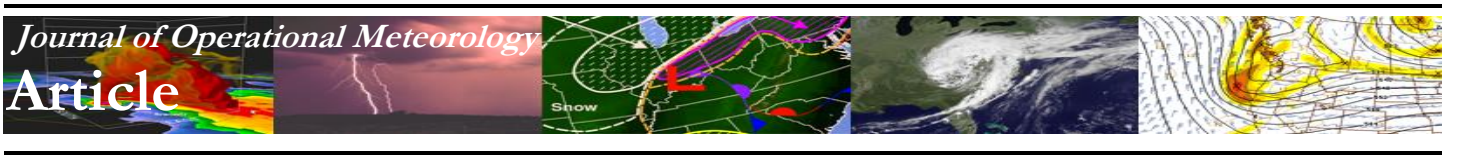

\title{
Principles and Applications of Dual-Polarization Weather Radar. Part III: Artifacts
}

\author{
MATTHEW R. KUMJIAN \\ Advanced Study Program, National Center for Atmospheric Research*, Boulder, Colorado
}

(Manuscript received 22 April 2013; review completed 7 August 2013)

\begin{abstract}
With the new data collected with polarimetric radars comes a set of new data quality issues and artifacts. It is important for these artifacts to be recognized and understood as such, thereby allowing operational meteorologists to focus on the interpretation of the physically important observations. In this third part of the series, artifacts found in polarimetric radar data are described and explained. These include attenuation and differential attenuation, nonuniform beam filling, depolarization streaks, and three-body scattering signatures. Examples of each are given, along with explanations of what they mean, and how they may be used to provide some information about a storm and its microphysics.
\end{abstract}

\section{Introduction}

Polarimteric radar data offer important new information regarding the type and size of precipitation particles within storms, as shown in the first two parts of this series (Kumjian 2013a,b). However, with this new technology comes a new collection of possible data artifacts. Proper identification and understanding of these potential problems will alleviate confusion in the interpretation and utilization of dual-polarization data. This paper discusses some of the most common artifacts present in dual-polarization data, their effect on data quality, and their possible causes.

\section{Artifacts}

\section{a. Attenuation and differential attenuation}

Attenuation is the reduction in power of the transmitted radar signal as it propagates through a medium (e.g., rain and hail). Power is removed from the propagating signal and dissipated as thermal energy within the hydrometeors (absorption) or scattered away from the particle in directions other than parallel or antiparallel to the direction of propagation (scattering). The specific attenuation of the horizontally polarized signal $\left(A_{H}\right)$, given in units of power loss per unit radial distance, or $\mathrm{dB} \mathrm{km}^{-1}$, is the amount (per $\mathrm{km})$ that the radar reflectivity at hori- zontal polarization $\left(Z_{H}\right)$ decreases owing to signal extinction by scattering and/or absorption. It is dependent on the characteristics of the particles (e.g., size or relative permittivity) and the particle size distribution, and is inversely proportional to the radar wavelength. This inverse dependence on radar wavelength means that higher-frequency (shorter-wavelength) radar signals suffer from more attenuation in rain than lowerfrequency (longer-wavelength) systems.

Similar to specific attenuation, specific differential attenuation $\left(A_{\mathrm{DP}}\right)$ is the difference in attenuation between the horizontally and vertically polarized channels. Analogous to attenuation causing a decrease in $Z_{H}$ and $Z_{V}$, differential attenuation most often causes a decrease in the differential reflectivity $\left(Z_{\mathrm{DR}}\right)$. Therefore, $A_{\mathrm{DP}}$ is the amount of decrease in $Z_{\mathrm{DR}}$ per $\mathrm{km}$. Note that, unlike $Z_{\mathrm{DR}}, A_{\mathrm{DP}}$ is proportional to the number concentration of particles within the radar sampling volume.

The Weather Surveillance Radar-1988 Doppler (WSR-88D) radar network operates at $\mathrm{S}$ band, or at a wavelength of about $10 \mathrm{~cm}$, so attenuation and differential attenuation typically are not major concerns. However, it can be observed in some cases, such as when the beam propagates through heavy precipitation cores in supercell storms (Fig. 1), or

\footnotetext{
*The National Center for Atmospheric Research is sponsored by the National Science Foundation. Corresponding author address: Dr. Matthew R. Kumjian, NCAR, P.O. Box 3000, Boulder, CO 80307 E-mail: kumjian@psu.edu
} 


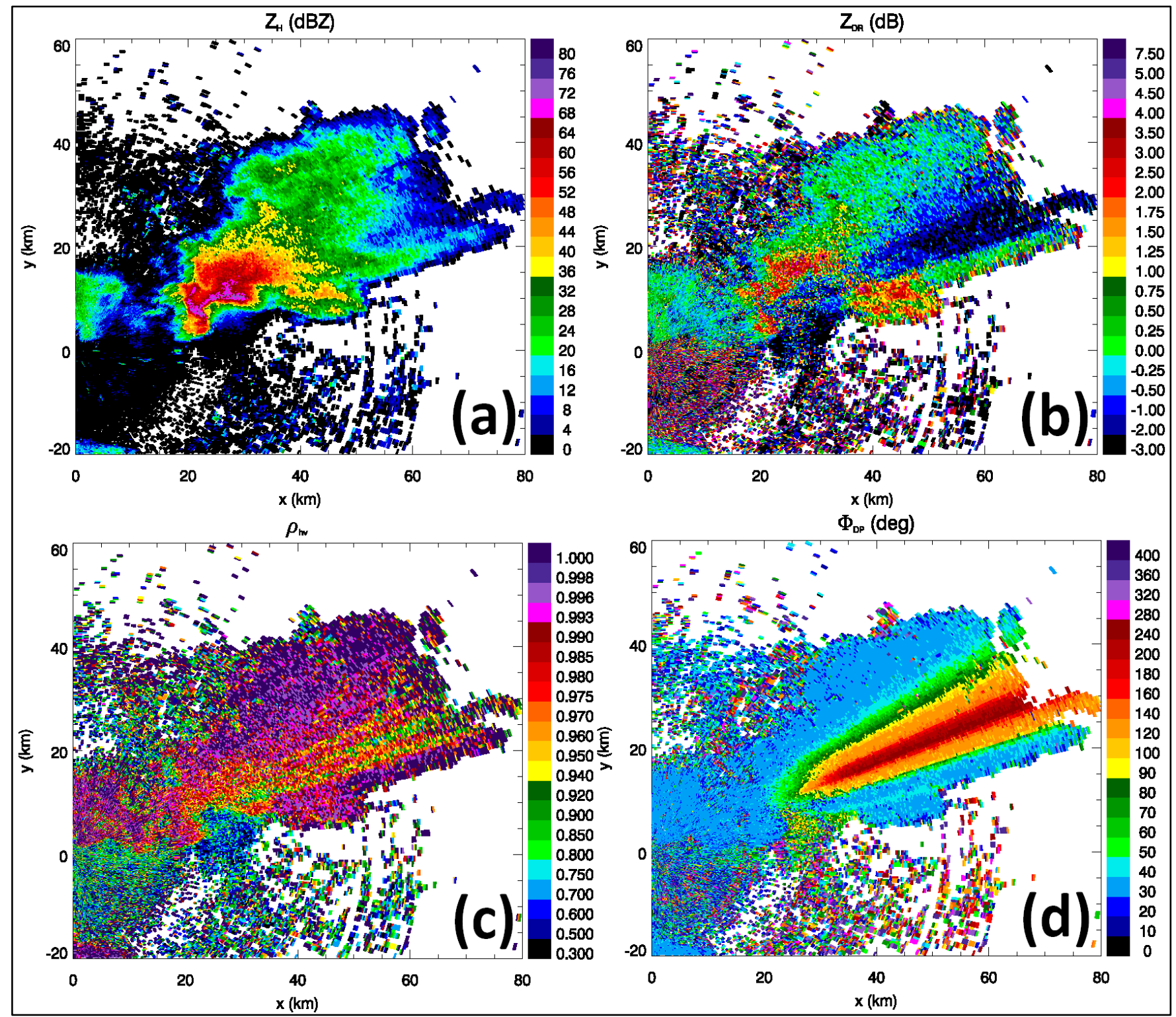

Figure 1. Four-panel display of the fields of (a) $Z_{H}$, (b) $Z_{\mathrm{DR}}$, (c) $C C$ or $\rho_{\mathrm{hv}}$, and (d) $\Phi_{\mathrm{DP}}$ from the dual-polarization WSR-88D radar in Hytop, AL (KHTX). Data are from the $0.5^{\circ}$ PPI scan taken at 2254 UTC 2 March 2012. Level-II data are used so that $\Phi_{\mathrm{DP}}$ may be presented. Note the anomalous differential attenuation downrange of the core, where $Z_{\mathrm{DR}}$ values drop to $<-3 \mathrm{~dB}$. Click image for an external version; this applies to all figures hereafter.

through the long axis of linear mesoscale convective systems (e.g., Ryzhkov and Zrnić 1995). Differential attenuation is evident in the negative $Z_{\mathrm{DR}}$ values downrange of the core. Notice also the increased differential propagation phase $\left(\Phi_{\mathrm{DP}}\right)$ in this area (Fig.1d); radials suffering from attenuation or differential attenuation often also exhibit large $\Phi_{\mathrm{DP}}$. Because phase measurements are unaffected by attenuation, $\Phi_{\mathrm{DP}}$ is extremely useful for correction of attenuation and differential attenuation (e.g., Bringi et al. 1990; Testud et al. 2000; Snyder et al. 2010;
Borowska et al. 2011; Gu et al. 2011). Indeed, $\Phi_{\mathrm{DP}}$ is used for correction of attenuation and differential attenuation in the pre-processing of polarimetric WSR88D radar moments, which are then used in the various algorithms (see Part II).

Attenuation or loss of the signal power is caused by some combination of absorption and scattering of the microwave radiation by hydrometeors. Absorption is dominant for small hydrometeors (i.e., those with diameters small compared to the radar wavelength). On the other hand, losses owing to scattering are 
dominant for large particles and are heavily impacted by resonance scattering effects. Whereas the intrinsic $A_{H}$ of hail is much higher than of rain, the intrinsic $A_{\mathrm{DP}}$ does not increase dramatically with hail size (e.g., Ryzhkov et al. 2013). Thus, we can expect that the smaller melting particles and large raindrops - that occur in much larger concentrations than large hailstones (assuming some sort of inverse exponential size distribution) - are the dominant contributors to $A_{\mathrm{DP}}$ in many cases. Using the model of Ryzhkov et al. (2013), in the case of melting hail we may separate the contributions of different hail sizes to the polarimetric radar variables (Fig. 2). As expected, whereas $A_{H}$ has the largest contributions from the largest hailstones, $A_{\mathrm{DP}}$ is dominated by the smaller particle sizes. Also note that $Z_{H}$ is dominated by the largest particles, whereas the majority of the contributions to $K_{\mathrm{DP}}$ come from smaller melting hail and raindrops (Fig. 2b).

\section{b. Nonuniform beam filling}

The region of differential attenuation in Fig. 1 also is coincident with a radially oriented reduction in the co-polar correlation coefficient $\left[\rho_{\text {hv }} ;\right.$ correlation coefficient $(C C)$ in the operational community]. Though often confused with attenuation ${ }^{1}$, this artifact is a result of nonuniform beam filling (NBF). Beam broadening with range can lead to inhomogeneous filling of the sampling volume (Fig. 3). In the event that there are large cross-beam gradients of $\Phi_{\mathrm{DP}}$ within the radar sampling volume, $\rho_{\text {hv }}(C C)$ is reduced (e.g., Ryzhkov 2007). This reduction in $\rho_{\mathrm{hv}}(C C)$ occurs because of the spread or diversity of $\Phi_{\mathrm{DP}}$ values within the sampling volume, which is analogous to the reduction in $\rho_{\mathrm{hv}}(C C)$ associated with the presence of resonance scatterers that produce nonzero differential phase shift upon backscatter $(\delta)$. Recall that a diversity of such phase shifts within the sampling volume reduces the $\rho_{\mathrm{hv}}(C C)$. In addition, large cross-beam gradients in $Z_{H}$ or $Z_{V}$ can lead to biases in $Z_{\mathrm{DR}}, \Phi_{\mathrm{DP}}$, and $C C$ (Ryzhkov 2007). Such cross-beam gradients can be in the azimuthal or elevation direction, though frequently occur in the elevation direction. This is because the beam may transect the melting layer, whereupon the top portion of the beam is filled with ice hydrometeors while the bottom portion of the beam is filled with melting or melted particles characterized by dramatically larger $K_{\mathrm{DP}}$ (Fig. 3).

\footnotetext{
${ }^{1}$ Recall that $C C$ or $\rho_{\mathrm{hv}}$ is not affected by attenuation; see Part I.
}

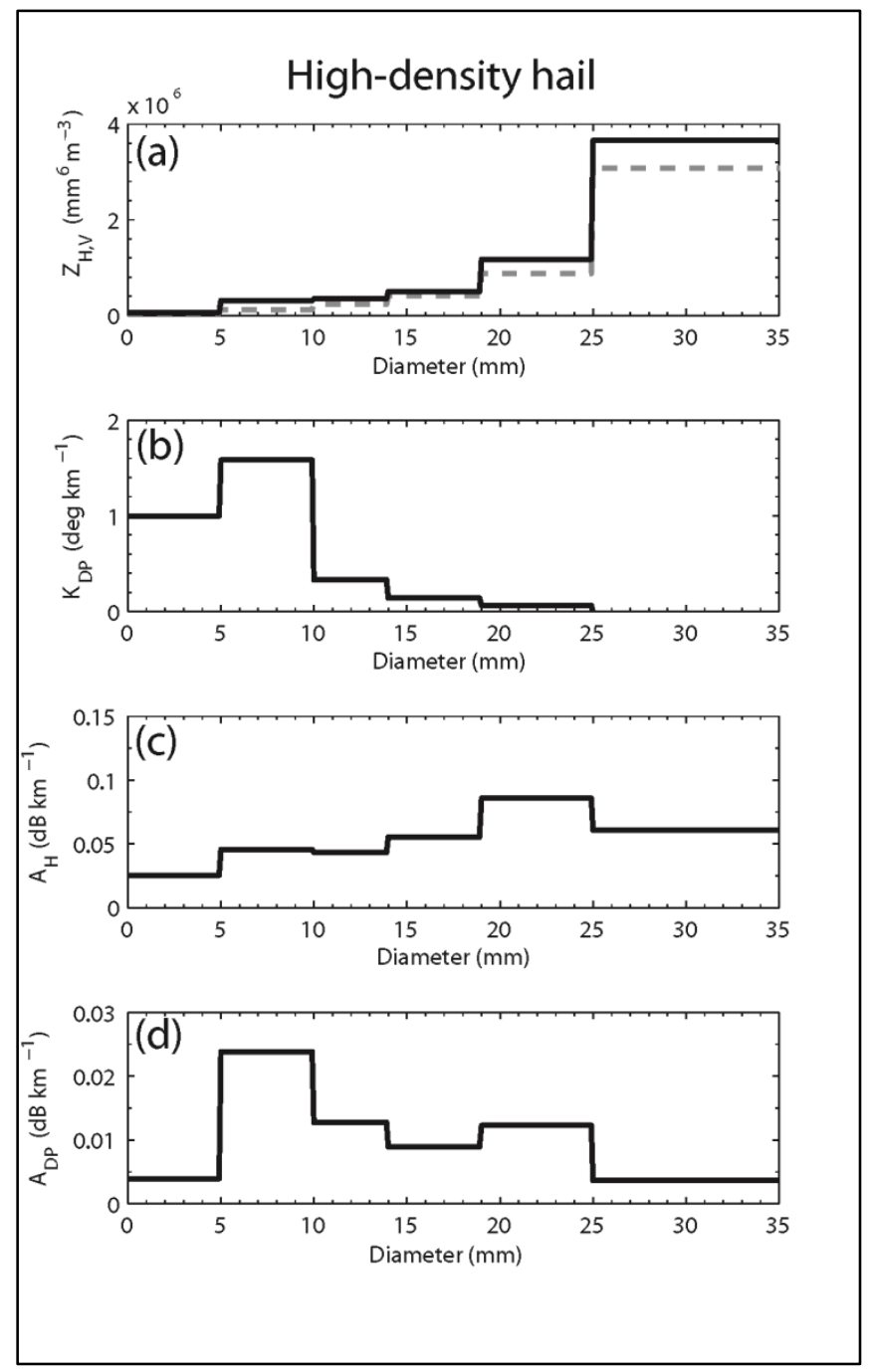

Figure 2. Relative contributions to (a) $Z_{H}$ and $Z_{V}$ (black and gray dashed lines, respectively), (b) $K_{\mathrm{DP}}$, (c) $A_{H}$, and (d) $A_{\mathrm{DP}}$, from different sizes of melting hailstones and raindrops, simulated from the model of melting hail of Ryzhkov et al. (2013). A biexponential distribution of high-density hail (i.e., the hailstones are composed of solid ice) is prescribed aloft at $4 \mathrm{~km}$ height, with a maximum hail size of $3.5 \mathrm{~cm}$. Calculations are for ground level.

NBF is common when convective storms are sampled at large distances from the radar and/or when radars operating with relatively large beamwidths are used to sample convective storms. The reduction in $\rho_{\text {hv }}$ causes increased statistical fluctuations in all polarimetric variables. The reduced data quality leads to deteriorated performance of automated algorithms developed for quantitative precipitation estimation and hydrometeor classification (see the example in Part II). The latest version of the hydrometeor classification algorithm (Park et al. 2009) accounts for regions of reduced signal quality, but meteorologists should be 


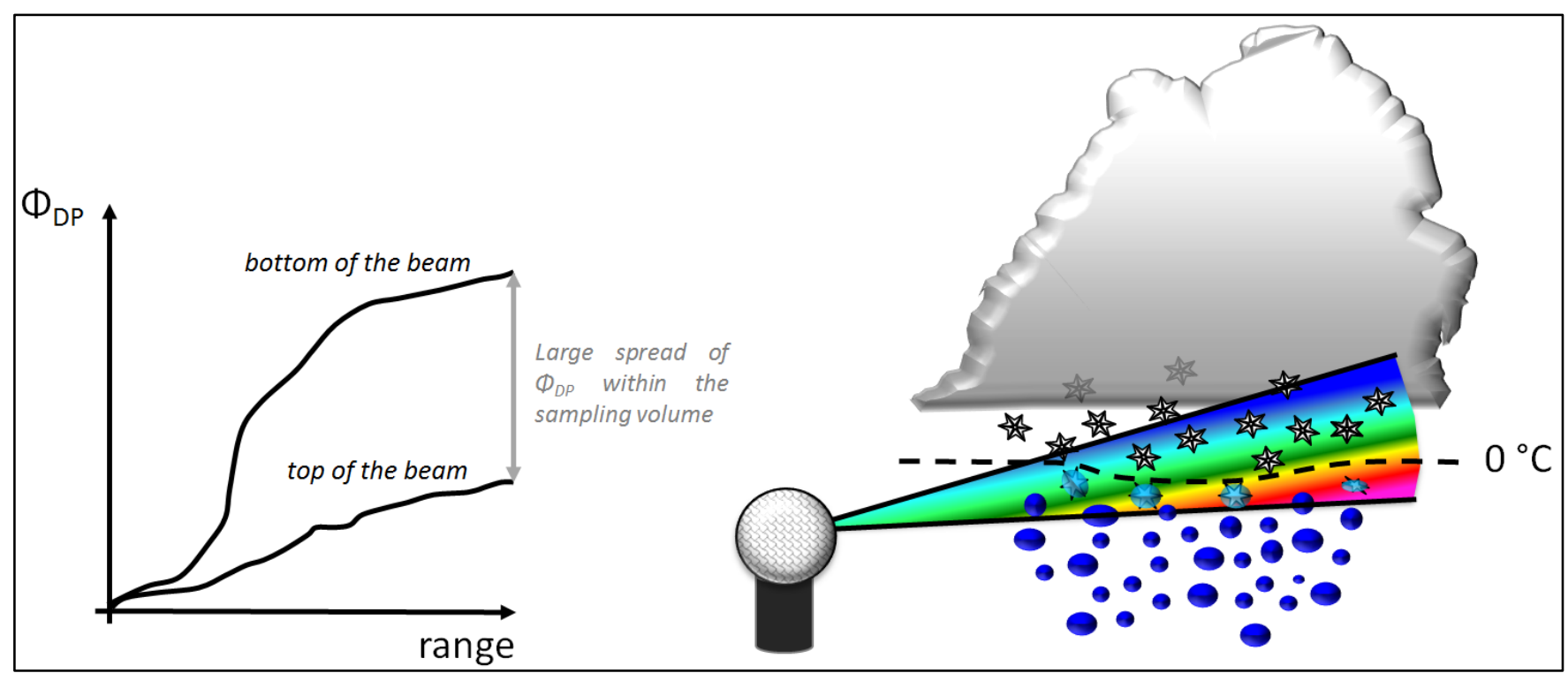

Figure 3. Schematic illustrating nonuniform beam filling. In this example, the bottom portion of the beam intercepts melting snow and heavy rain characterized by large $\Phi_{\mathrm{DP}}$ values (represented by shading with warmer colors) while the top of the beam intercepts ice-phase particles (with low $\Phi_{\mathrm{DP}}$, represented by shading with cooler colors) above the freezing level. This large spread of $\Phi_{\mathrm{DP}}$ results in a reduction of $\rho_{\mathrm{hv}}$ or $C C$. The figure was inspired by one from a National Weather Service (NWS) Warning Decision Training Branch module.

aware of these areas when interpreting polarimetric radar observations.

\section{c. Depolarization streaks}

In the presence of strong electric fields in the upper regions of storms, small ice crystals may align with the electric field vector. This phenomenon has been observed with polarimetric radars that transmit circularly polarized waves (e.g., Hendry and McCormick 1976; Kreihbel et al. 1996) and linearly polarized waves (e.g., Caylor and Chandrasekar 1996; Metcalf 1997; Ryzhkov and Zrnić 2007; Hubbert et al. 2010b). The common alignment of the crystals lasts until a lightning discharge substantially reduces the electric field intensity, whereupon the crystals return to more typical orientations (generally, with their larger dimension more-or-less aligned in the horizontal). Crystals aligned in an electric field that is neither purely horizontal or vertical can produce a peculiar artifact in polarimetric measurements by radars operating in the simultaneous transmission and reception mode, such as the WSR-88D radars (Ryzhkov and Zrnić 2007; Hubbert et al. 2010a,b; Zrnić et al. 2010a). Polarimetric radars operating in a mode of alternating transmission and reception are immune to this type of artifact.

The artifact appears as radial "streaks" of positive or negative $Z_{\mathrm{DR}}$ (e.g., Fig. 4), generally at higher elevation angle scans where the radar is sampling ice hydrometeors. These depolarization streaks are not visible in the $\rho_{\text {hv }}(C C)$ field, but are sometimes coincident with discernible increases in $\Phi_{\mathrm{DP}}$ (because the ice crystals are nonspherical). A necessary condition for these streaks to appear is a nonzero phase $\psi=\Phi_{t}+\phi_{d p} / 2$, where $\Phi_{t}$ is the system differential phase upon transmission and $\phi_{d p}$ is the intrinsic differential phase shift along the propagation path leading to the oriented crystals. In many cases, the transmitted wave propagates through rain at lower altitudes before entering the ice-phase region, acquiring nonzero $\phi_{d p}$, so this condition often is satisfied. The example in Fig. 4 shows that the depolarization streaks originate above the melting layer, in regions of strongly enhanced $K_{\mathrm{DP}}$, which indicates a large ice crystal mass content (see Part II). Indeed, lightning and thunder were reported shortly after the depolarization streaks appeared (J. Picca 2013, personal communication).

When the transmitted wave enters the so-called "depolarizing medium" (i.e., the oriented ice crystals, or those canted with non-zero mean canting angle), the electromagnetic wave becomes progressively depolarized. This also is known as cross-coupling of the $\mathrm{H}$ and $\mathrm{V}$ components of the transmitted wave. Recall that in the Rayleigh scattering approximation, a hydrometeor can be modeled as a spheroid with electric dipoles aligned with its major and minor axes. If the polarization vector of the radar wave has a 


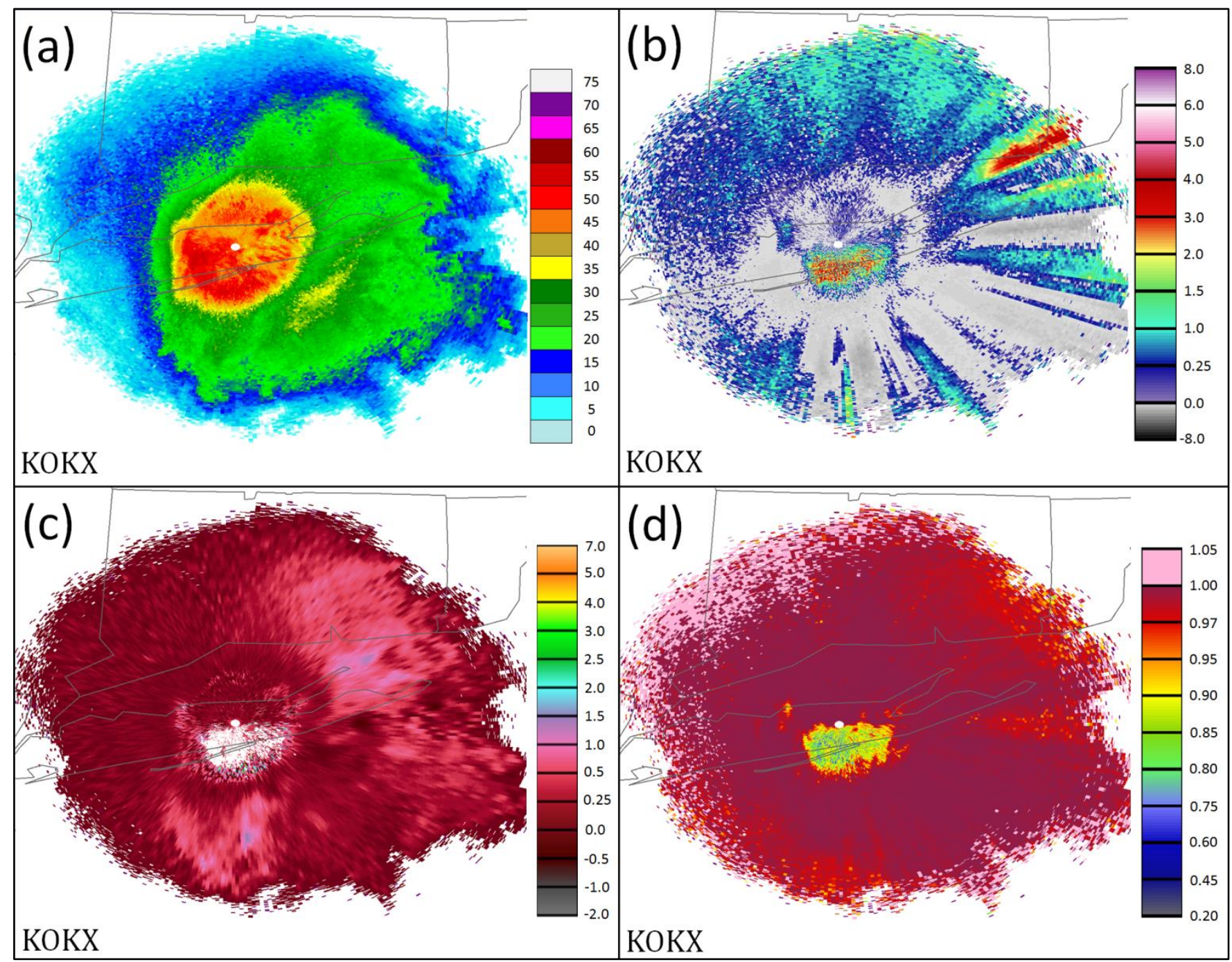

Figure 4. Example of depolarization streaks in the field of $Z_{\mathrm{DR}}$, from a Nor'Easter observed with the polarimetric WSR-88D radar in Upton, NY (KOKX) at 2355 UTC 8 February 2013. Fields shown are (a) $Z_{H}$, (b) $Z_{\mathrm{DR}}$, (c) $K_{\mathrm{DP}}$, and (d) $C C$ or $\rho_{\mathrm{hv}}$. Data were collected at the $3.4^{\circ}$ elevation angle. The depolarization streaks in $Z_{\mathrm{DR}}$ are particularly evident to the east and south of the radar.

component aligned with a particle's dipole (unless the polarization vector is perpendicular to that dipole), the dipole is excited and emits secondary radiation. Thus, in the general case when a hydrometeor is not perfectly aligned with its minor axis in the vertical, both of the hydrometeor's dipoles are excited when illuminated by either the $\mathrm{H}$ or $\mathrm{V}$ polarization radar signal (Figs. 5a,b). These excited dipoles then emit secondary radiation that can be resolved into components with $\mathrm{H}$ and $\mathrm{V}$ polarization (Figs. 5c,d).

Depolarization is said to occur if a particle, when illuminated with an electromagnetic wave of one polarization, scatters radiation with a component of the orthogonal polarization (Fig. 5). Once the signal is depolarized, the remainder of the data downrange of the point of depolarization are compromised. Such diminished data quality inhibits the usefulness of $Z_{\mathrm{DR}}$ for quantitative precipitation estimation and hydrometeor classification. However, these depolarization streaks, though a detriment to quantitative precipitation estimation and hydrometeor classification, can serve as a useful indicator of the presence of a relatively strong electric field (i.e., sufficiently strong to orient low-inertia crystals). Though the presence of such streaks does not always indicate an imminent lightning discharge, further research may determine their applicability as a lightning forecasting tool. Additionally, the generation of electric charge often requires rimed particles, implying the presence of supercooled liquid water in the vicinity of where the streaks first appear along the radial. Localization of areas of ongoing riming - especially in embedded convection 


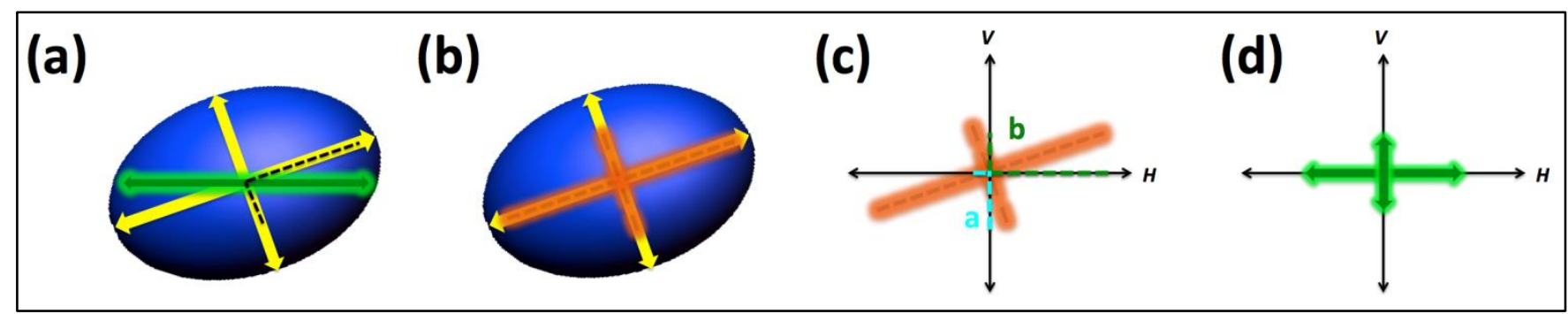

Figure 5. Cartoon illustrating the process of signal depolarization for a canted raindrop. (a) A canted raindrop, with dipoles along the major and minor axes given by the yellow arrows, is illuminated by an incident electromagnetic wave with horizontal polarization (green arrow). Because the incident polarization vector can be decomposed into components along each axis of the particle (dashed black lines along the particle axes), (b) dipoles along each axis are excited and scatter radiation, represented by the orange highlighting. (c) The backscattered radiation from both illuminated dipoles can be decomposed into the horizontal and vertical polarization directions, given by the green and light blue dashed lines. (d) Thus, the backscattered radiation has components of both horizontal and vertical polarizations (green arrows).

within more widespread stratiform precipitation-may be a useful feature to indicate conditions favorable for aircraft icing. These and other applications of depolarization streaks remain to be investigated in future research.

Also note that cross-coupling as a result of antenna polarization errors may introduce biases in $Z_{\mathrm{DR}}$ in rain when $\phi_{d p}$ has accumulated significantly $\left(>50^{\circ}\right.$; Hubbert et al. 2010a,b). In such a case, there will be no clear manifestation such as depolarization streaks. If biases become large enough, quantitative precipitation estimates that depend on $Z_{\mathrm{DR}}$ or a hydrometeor classification that uses $Z_{\mathrm{DR}}$ as an input may be affected negatively. However, Zrnić et al. (2010a) found that these $Z_{\mathrm{DR}}$ biases are relatively small. It remains to be seen how large of an impact this potential source of error will be in the upgraded WSR-88D radar network.

\section{d. Polarimetric three-body scattering signature}

The three-body scattering signature (TBSS; Zrnić 1987; Wilson and Reum 1988) in the $Z_{H}$ field has been used to indicate the presence of hail (e.g., Lemon 1998). The signature appears as a radially oriented "spike" of weak $Z_{H}$ protruding from the far side (relative to the radar) of the storm. It occurs when electromagnetic radiation scattered off hailstones reflects off the ground, then scatters again off hailstones back towards the radar. In polarimetric radar observations, the near-storm portion of the TBSS often is observed to have extremely large $Z_{\mathrm{DR}}$ values $(>6 \mathrm{~dB})$ and very low $C C(<0.5$; see Fig. 6$)$. Farther downrange, $Z_{\mathrm{DR}}$ values become negative and $\rho_{\mathrm{hv}}$ values remain very low (e.g., Hubbert and Bringi 2000).

The radially oriented spike of very low $\rho_{\text {hv }}$ or $C C$ down radial of the hail core is the easiest way to detect a polarimetric TBSS (PTBSS). Sometimes, the en- hancement of $Z_{\mathrm{DR}}$ may look like a $Z_{\mathrm{DR}}$ column. The cause of the large $Z_{\mathrm{DR}}$ associated with the PTBSS can be explained within the framework of simple scattering theory. The received $Z_{\mathrm{DR}}$ (in linear scale) from the PTBSS can be considered the product of three factors, as explained by Picca and Ryzhkov (2012):

$Z_{d r} \approx \frac{P_{H}}{P_{V}} \times \frac{\sigma_{H}}{\sigma_{V}} \times \frac{L_{H}}{L_{V}}$.

The first factor $\left(P_{H} / P_{V}\right)$ characterizes the difference between radiation patterns of the scatterer at $\mathrm{H}$ and $\mathrm{V}$ polarizations. The second factor $\left(\sigma_{\mathrm{H}} / \sigma_{\mathrm{V}}\right)$ is the ratio between radar cross sections (at $\mathrm{H}$ and $\mathrm{V}$ polarizations) of the ground or underlying surface beneath the hail core. The third factor is a ratio of attenuation factors at $\mathrm{H}$ and $\mathrm{V}$ polarization $\left(\mathrm{L}_{\mathrm{H}} / \mathrm{L}_{\mathrm{V}}\right)$ which characterizes losses attributable to propagation through the hailstones as well as scatter off the ground or underlying surface. Note that each of these factors is a function of the angle $\theta$ of the radiation path between the hail core and the ground (measured from nadir, or from the downward vertical direction).

In general, the radiation patterns at $\mathrm{H}$ and $\mathrm{V}$ polarizations are different. The radiation pattern of an electric dipole has a null along the axis of that dipole. Consider a perfectly oriented hailstone with its minor axis aligned in the vertical, and its major axis aligned in the horizontal. If the dipole along the hailstone's minor axis becomes excited, it does not radiate in the upward or downward directions. In contrast, the dipole along the hailstone's major axis does radiate in the downward and upward directions (Fig. 7i). Thus, radiation scattered downwards by this perfectly aligned hailstone has no vertically polarized component, resulting in infinite $Z_{\mathrm{DR}}$ (Kumjian et al. 2010). 


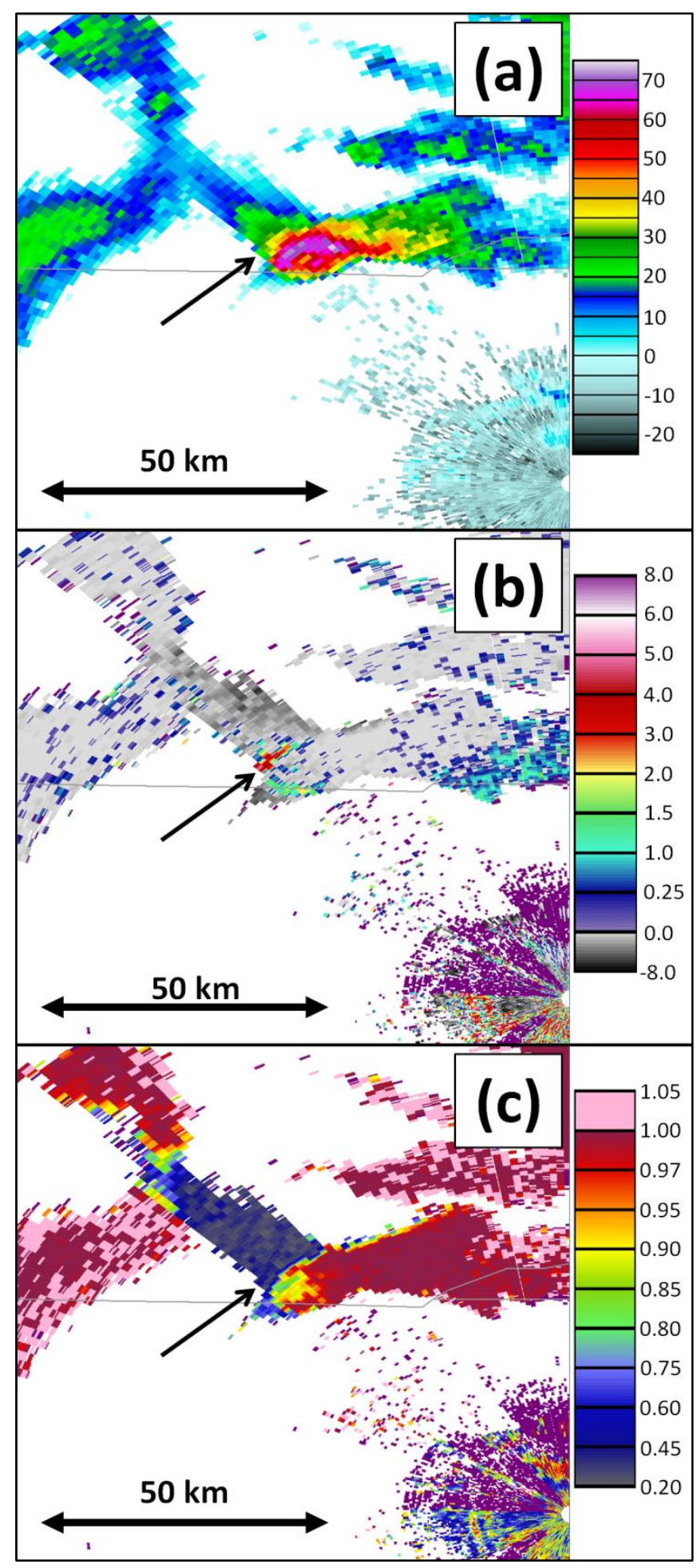

Figure 6. Example of a spectacular three-body scattering signature downrange from a hail core, observed with the polarimetric WSR88D radar near Knoxville, TN (KMRX). Variables shown are (a) $Z_{H}$, (b) $Z_{\mathrm{DR}}$, and (c) $\rho_{\mathrm{hv}}$ or $C C$. Data collected at 2318 UTC 2 March 2012 at the $2.4^{\circ}$ elevation angle. Note the high $Z_{\mathrm{DR}}$ immediately downrange of the hail core (indicated by the arrow), followed by negative $Z_{\mathrm{DR}}$ values (and collocated with extremely low $C C$ or $\rho_{\text {hv }}$ ) farther downrange.
In reality, of course, the PTBSS is produced by downscatter paths over a conical region (e.g., Zrnić 1987; Fig. 7). Thus, the V-polarized component of the downscattered signal is nonzero, though still much smaller than the H-polarized component. This produces large (but finite) bistatic $Z_{\mathrm{DR}}$ (e.g., Aydin et al. 1998). In contrast, at these downscattered paths in directions close to straight down, the ratio of ground cross sections $\left(\sigma_{\mathrm{H}} / \sigma_{\mathrm{V}}\right)$ is close to one (Fig. 7ii), assuming the absence of any interesting geological features such as mountains or valleys. Similarly, the loss factor $\mathrm{L}_{\mathrm{H}} / \mathrm{L}_{\mathrm{V}}$ is also near unity (Fig. 7iii). Thus, the PTBSS $Z_{\mathrm{DR}}$ is determined primarily by the factor $\mathrm{P}_{\mathrm{H}} / \mathrm{P}_{\mathrm{V}}$, which is expected to be very large for the reasons explained above. Because the downscattered paths at angles near nadir are shorter than those at larger angles, the highest $Z_{\mathrm{DR}}$ (and lowest $\rho_{\mathrm{hv}}$ ) are expected to be located nearest the hail core. Indeed, observations of PTBSSs reveal that the highest $Z_{\mathrm{DR}}$ signatures are separated from the presumed hail core (maximum in $Z_{H}$ ) by ranges comparable to the height above ground level of the sampled hail core (Kumjian et al. 2010). Extended TBSSs indicate contributions from downscattered paths at larger $\theta$. At such larger $\theta$, the bistatic $Z_{\mathrm{DR}}$ (that is, the ratio $\mathrm{P}_{\mathrm{H}} / \mathrm{P}_{\mathrm{V}}$ ) decreases (Aydin et al. 1998; right side of Fig. 7i). Similarly, the ratios $\mathrm{L}_{\mathrm{H}} / \mathrm{L}_{\mathrm{V}}$ and $\sigma_{\mathrm{H}} / \sigma_{\mathrm{V}}$ decrease with increasing $\theta$ (e.g., Ulaby et al. 1982; Hubbert and Bringi 2000; Picca and Ryzhkov 2012), often leading to negative $Z_{\mathrm{DR}}$ values.

The PTBSS, especially the reduced $\rho_{\text {hv }}$ or $C C$, can be especially useful for hail detection when the conventional $Z_{H}$ TBSS is obfuscated by the presence of other storms. However, the very large $Z_{\mathrm{DR}}$ values should not be mistaken for a $Z_{\mathrm{DR}}$ column. Though $Z_{\mathrm{DR}}$ columns can be coincident with reduced $\rho_{\text {hv }}$ or $C C$, the very low nonmeteorological $\rho_{\mathrm{hv}}$ or $C C$ values associated with the PTBSS can be used to discern between the two signatures. Also note that the PTBSS only appears on the rear side of the storm (i.e., the downrange side). It is unlikely that the PTBSS can be used as an indicator of hail size, just as the TBSS in $Z_{H}$ is ambiguous (Zrnić et al. 2010b). The explanation above considered hailstone radiation patterns in the Rayleigh approximation. Even at $\mathrm{S}$ band, hailstones larger than about $2-3 \mathrm{~cm}$ in diameter are outside of the valid limits of the Rayleigh approximation, in which case their radiation patterns are far more complex than those produced by the simple dipole structure. Thus, though larger hailstones may be able to downscatter more radiation, their intrinsic bistatic $Z_{\mathrm{DR}}$ is much lower than for the smaller hailstones (that have radi- 


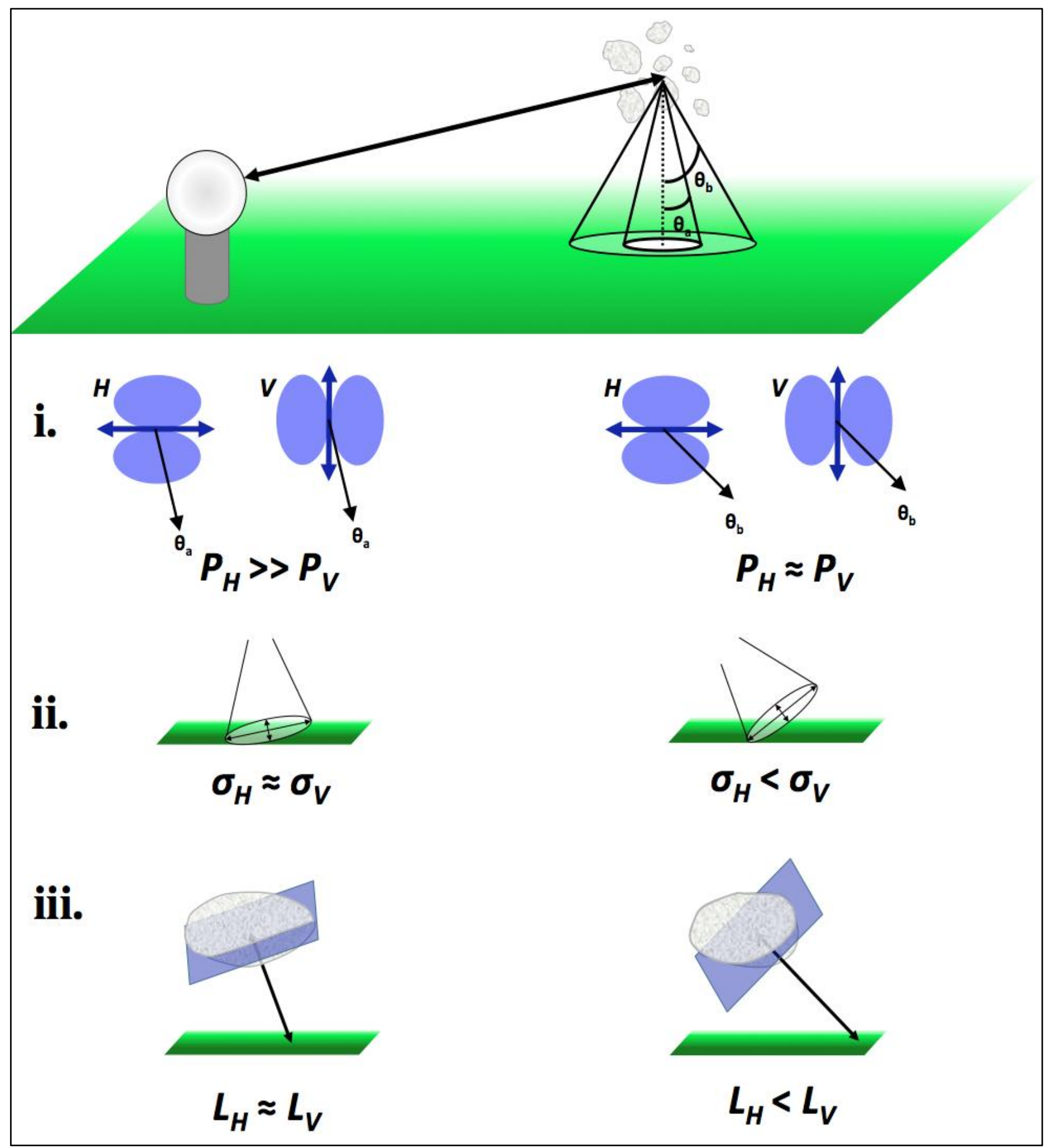

Figure 7. Schematic illustrating factors contributing to the polarimetric TBSS. In the top panel, example propagation paths of electromagnetic radiation are shown. Radiation scatters off the hailstones and toward the ground at two off-nadir angles $\left(\theta_{\mathrm{a}}\right.$ and $\left.\theta_{\mathrm{b}}\right)$. The measured $Z_{\mathrm{DR}}$ in the PTBSS is the product of three ratios: $i$. The "bistatic $Z_{\mathrm{DR}}$ " (Aydin et al. 1998), which is the ratio of the powers of the radiation scattered downward by the hailstones $\left(\mathrm{P}_{\mathrm{H}} / \mathrm{P}_{\mathrm{V}}\right)$; ii. The ratio of the ground backscattering cross section at $\mathrm{H}$ and $\mathrm{V}$ polarizations $\left(\sigma_{\mathrm{H}}\right.$ $/ \sigma_{\mathrm{V}}$ ); and iii. A factor representing the differential attenuation suffered by the signal as it propagates through the hailstones and off the ground $\left(\mathrm{L}_{\mathrm{H}} / \mathrm{L}_{\mathrm{V}}\right)$. The left column schematically shows these three factors for $\theta_{\mathrm{a}}$, close to nadir, whereas the right column shows the three factors for $\theta_{\mathrm{b}}>\theta_{\mathrm{a}}$. Adapted from Picca and Ryzhkov (2012), with changes. 
ation patterns more closely approximated by two dipoles aligned with their principal axes), as seen in Fig. 8.

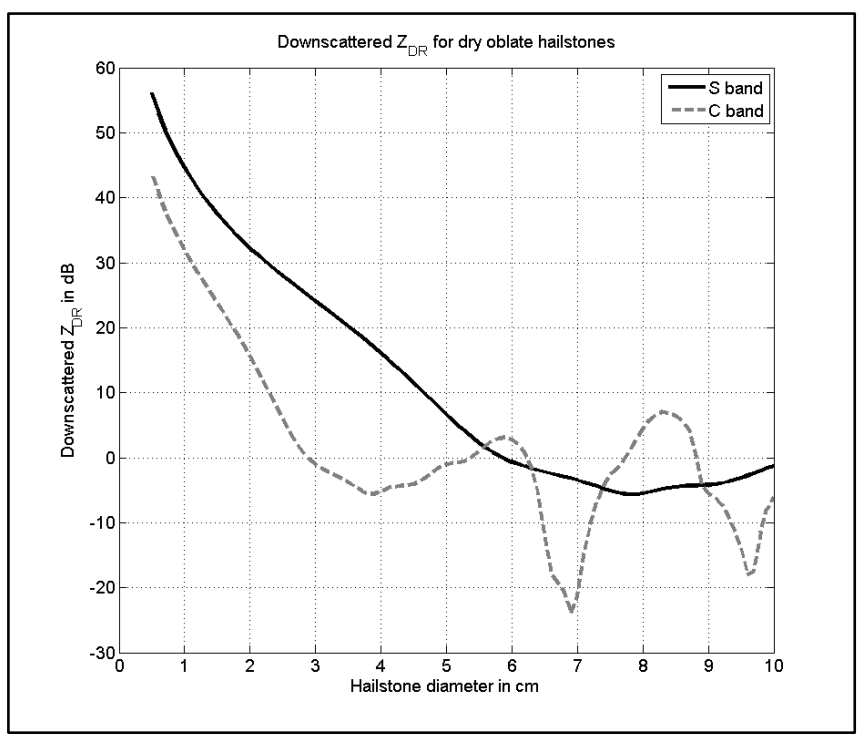

Figure 8. Downscattered or bistatic $Z_{\mathrm{DR}}$ produced by dry, oblate hailstones with an axis ratio of 0.8 for $\mathrm{S}$ and $\mathrm{C}$ bands (black solid and gray dashed lines, respectively). From Kumjian et al. (2010). Note that the anomalously high $Z_{\mathrm{DR}}$ observed in PTBSSs is more efficiently produced by bistatic scattering from smaller hailstones.

\section{Discussion and conclusions}

With the new influx of dual-polarization radar data will come a new set of artifacts and data quality issues with which radar users should be familiar. Such artifacts reviewed in this paper are attenuation and differential attenuation, nonuniform beam filling, depolarization streaks, and the polarimetric three-body scatter signature. These artifacts have a negative effect on data quality for use in automated algorithms, but may provide some useful information about the conditions in the storm leading to the artifact's appearance. For example, precipitation cores producing differential attenuation may disrupt quantitative precipitation estimates downrange of the core, but do provide direct evidence of extremely heavy precipitation and likely melting hail within the core. Depolarization streaks may alert operational meteorologists to the presence of a strong electric field and possible lightning production. And, the polarimetric three-body scattering signature observed aloft reveals the presence of hail, providing some lead time to the onset of hail at the surface.

Understanding these data artifacts will improve interpretation of the radar data and provide insight into conditions within the storms. In addition, understanding the regions for which data quality may be compromised is critical for the most efficient and effective use of dual-polarization radar products generated by automated algorithms, which should aid in short-term forecasts and warning decisions.

Acknowledgments. Useful discussions with Drs. Alexander Ryzhkov (Cooperative Institute for Mesoscale Meteorological Studies, CIMMS)/(National Severe Storms Laboratory, NSSL) and Dušan Zrnić (NSSL) are acknowledged, as are those with Mr. Joey Picca (NWS New York). Dr. John Hubbert (NCAR), Scott Ganson (NWS Radar Operations Center), Joey Picca, Professor Paul Smith (South Dakota School of Mines and Technology), Paul Schlatter (NWS Program Coordination Office), and Dr. Matt Bunkers (NWS Rapid City) are thanked for their reviews of - and insightful comments on - the manuscript. Jon Zeitler (NWS Austin/San Antonio) provided the technical editing for this series. Support for the author comes from the National Center for Atmospheric Research (NCAR) Advanced Study Program. NCAR is sponsored by the National Science Foundation.

\section{REFERENCES}

Aydin, K., S. H. Park, and T. M. Walsh, 1998: Bistatic dualpolarization scattering from rain and hail at $\mathrm{S}$ - and Cband frequencies. J. Atmos. Oceanic Technol., 15, $1110-1121$.

Borowska, L., A. V. Ryzhkov, D. S. Zrnić, C. Simmer, and R. D. Palmer, 2011: Attenuation and differential attenuation of 5 -cm-wavelength radiation in melting hail. J. Appl. Meteor. Climatol., 50, 59-76.

Bringi, V. N., V. Chandrasekar, N. Balakrishnan, and D. S. Zrnić, 1990: An examination of propagation effects on radar measurements at microwave frequencies. $J$. Atmos. Oceanic Technol., 7, 829-840.

Caylor, I. J., and V. Chandrasekar, 1996: Time-varying crystal orientation in thunderstorms observed with multiparameter radar. IEEE Trans. Geosci. Remote Sens., 34, 847-858.

Gu, J.-Y., A. V. Ryzhkov, P. Zhang, P. Neilley, M. Knight, B. Wolf, an D.-I. Lee, 2011: Polarimetric attenuation correction in heavy rain at $\mathrm{C}$ band. J. Appl. Meteor. Climatol., 50, 39-58.

Hendry, A., and G. C. McCormick, 1976: Radar observations of alignment of precipitation particles by electrostatic fields in thunderstorms. J. Geophys. Res., 81, 5353-5357.

Hubbert, J. C., and V. N. Bringi, 2000: The effects of threebody scattering on differential reflectivity signatures. $J$. Atmos. Oceanic Technol., 17, 51-61. 
, S. M. Ellis, M. Dixon, and G. Meymaris, 2010a: Modeling, error analysis, and evaluation of dualpolarization variables obtained from simultaneous horizontal and vertical polarization transmit radar. Part I: Modeling and antenna errors. J. Atmos. Oceanic Technol., 27, 1583-1598. , and _, 2010b: Modeling, error analysis, and evaluation of dual-polarization variables obtained from simultaneous horizontal and vertical polarization transmit radar. Part II: Experimental data. J. Atmos. Oceanic Technol., 27, 1599-1607.

Krehbiel, P. R., T. Chen, S. McCrary, W. Rison, G. Gray, and M. Brook, 1996: The use of dual-channel circularpolarization radar observations for remotely sensing storm electrification. Meteor. Atmos. Phys., 59, 65-82.

Kumjian, M. R., 2013a: Principles and applications of dualpolarization radar. Part I: description of the polarimetric radar variables. J. Operational Meteor., 1 (19), 226242.

, 2013b: Principles and applications of dualpolarization radar. Part II: Warm- and cold-season applications. J. Operational Meteor., 1 (20), 243-264.

, J. C. Picca, S. M. Ganson, A. V. Ryzhkov, and D. S. Zrnić, 2010: Three-body scattering signatures in polarimetric radar data. NOAA/NSSL report, $12 \mathrm{pp}$. [Available online at: www.nssl.noaa.gov/publications/ wsr88d_reports/FINAL TBSS.doc.]

Lemon, L. R., 1998: The radar "three-body scatter spike": An operational large-hail signature. Wea. Forecasting, 13, 327-340.

Metcalf, J. I., 1997: Temporal and spatial variations of hydrometeor orientation of hydrometeors in thunderstorms. J. Appl. Meteor., 36, 315-321.

Park, H. S., A. V. Ryzhkov, D. S. Zrnić, and K.-E. Kim, 2009: The hydrometeor classification algorithm for the polarimetric WSR-88D: Description and application to an MCS. Wea. Forecasting, 24, 730-748.

Picca, J. C., and A. V. Ryzhkov, 2012: A dual-wavelength polarimetric analysis of the 16 May 2010 Oklahoma City extreme hailstorm. Mon. Wea. Rev., 140, 13851403.
Ryzhkov, A. V., 2007: The impact of beam broadening on the quality of radar polarimetric data. J. Atmos. Oceanic Technol., 24, 729-744.

, and D. S. Zrnić, 1995: Precipitation and attenuation measurements at a $10-\mathrm{cm}$ wavelength. J. Appl. Meteor., 34, 2121-2134.

, and _ 2007: Depolarization in ice crystals and its effect on radar polarimetric measurements. J. Atmos. Oceanic Technol., 24, 1256-1267.

, M. R. Kumjian, S. M. Ganson, and A. P. Khain, 2013: Polarimetric characteristics of melting hail, Part I: Theoretical simulations using spectral microphysical modeling. J. Appl. Meteor. Climatol., in press.

Snyder, J. C., H. B. Bluestein, G. Zhang, and S. J. Frasier, 2010: Attenuation correction and hydrometeor classification of high-resolution, X-band, dual-polarized mobile radar measurements in severe convective storms. J. Atmos. Oceanic Technol., 27, 1979-2001.

Testud, J., E. Le Bouar, E. Obligis, and M. Ali-Mehenni, 2000: The rain profiling algorithm applied to polarimetric weather radar. J. Atmos. Oceanic Technol., 17, 332-356.

Ulaby, F., R. Moore, and A. Fung, 1982: Microwave remote sensing: Active and passive, Volume II: Surface scattering and emission theory. Addison-Wesley Publishing Company, 634 pp.

Wilson, J. W., and D. Reum, 1988: The flare echo: Reflectivity and velocity signature. J. Atmos. Oceanic Technol., 5, 197-205.

Zrnić, D. S., 1987: Three-body scattering produces precipitation signature of special diagnostic value. Radio Sci., 22, 76-86.

, R. J. Doviak, G. Zhang, and A. V. Ryzhkov, 2010a: Bias in differential reflectivity due to cross coupling through the radiation patterns of polarimetric weather radars. J. Atmos. Oceanic Technol., 27, 1624-1637.

, G. Zhang, V. Melnikov, and J. Andrić, 2010b: Threebody scattering and hail size. J. Appl. Meteor. Climatol., 49, 687-700. 